\title{
In reply: Iliac crest bone graft donor site analgesia: a new indication for erector spinae plane block
}

\author{
Ban C. H. Tsui, MD, FRCPC (1) • Vivian Ip, MBCHB - Rakesh V. Sondekoppam, MD
}

Received: 27 November 2018/ Accepted: 28 November 2018/Published online: 17 December 2018

(c) Canadian Anesthesiologists' Society 2018

\section{To the Editor,}

We read with great interest the comments by Gurkan et $a .^{1}{ }^{1}$ on our quadratus lumborum (QL) block article. ${ }^{2}$ Although Gurkan et al. ${ }^{1}$ refer to a higher pain score and narcotic consumption in our case series, two out of four patients had chronic pain and were on home opioids preoperatively, which might explain their inferior pain outcomes. The dermatomal spread in patients of our study receiving transmuscular QL (TQL) block was consistently between T9-L2, correlating with the anatomical findings. Despite this, we thank the authors for sharing their clinical observation as it aligns with our similar experience in using erector spinae plane (ESP) block to provide analgesia for iliac crest autograft harvest. In fact, we also recently encountered a comparable case in a pediatric chronic pain patient undergoing a repeat open reduction and internal fixation of tibial fracture and iliac crest autograft harvest. This patient was successfully managed with a multimodal analgesia regimen incorporating a combination of sciatic nerve and continuous lumbar ESP blocks. ${ }^{3}$

The anterior layer of the thoracolumbar fascia forms the anterior border of the QL, separating it from the psoas muscle anteriorly, while the middle layer of the fascia isolates the QL from the erector spinae muscle posteriorly. The ventral rami of the spinal nerves (L1-4), after exiting the intervertebral foramina, are encased within the psoas

B. C. H. Tsui, MD, FRCPC $(\bowtie)$.

Department of Anesthesiology, Perioperative and Pain Medicine, Stanford University School of Medicine, Stanford, CA, USA

e-mail: bantsui@stanford.edu

V. Ip, MBCHBR. V. Sondekoppam, MD

Department of Anesthesiology and Pain Medicine, University of Alberta, Edmonton, AB, Canada major muscle to form the lumbar plexus. The T12 nerve and L1 and L2 branches of the lumbar plexus pass through the anterior fascia of the QL muscle, which forms the basis of performing a TQL block. For other QL blocks, local anesthetic (LA) is deposited onto the lateral (i.e., QL-I) or posterior (i.e., QL-2), aspect of the QL muscle. In a lumbar ESP block, since the LA is injected between the erector spinae and the transverse process, there is a potential for the LA to spread onto the L1 and L2 dermatomes either via the lumbar paravertebral space (as seen with thoracic ESP) or by a lateral spread through the middle thoracolumbar fascia. This may, at least in part, explain the similar clinical effect seen between the TQL and ESP blocks. ${ }^{4,5}$ While cadaveric ESP injections in the thorax have shown conflicting results in terms of spread to the paravertebral space and/or the ventral ramus, little is known about the spread of lumbar ESP block injectates. Hence, it is necessary to explore the mechanism of lumbar ESP injections, and more anatomical and clinical trials are necessary to explore its utility.

Conflicts of interest None declared.

Editorial responsibility This submission was handled by Dr. Philip M. Jones, Associate Editor, Canadian Journal of Anesthesia.

\section{References}

1. Gurkan Y, Aksu C. Iliac crest bone graft donor site analgesia: a new indication for erector spinae plane block. Can J Anesth 2019; 66. DOI: https://doi.org/10.1007/s12630-018-01276-6.

2. Sondekoppam RV, Ip V, Johnston DF, et al. Ultrasound-guided lateral-medial transmuscular quadratus lumborum block for analgesia following anterior iliac crest bone graft harvesting: a clinical and anatomical study. Can J Anesth 2018; 65: 178-87. 
3. Darling C, Lin C, Caruso TJ, Tsui BC. Lumbar erector spine plane catheter via a thoracic approach for iliac chest autograft in a pediatric patient. J Clin Anesth 2019; 54: 164-165. DOI: https:// doi.org/10.1016/j.jclinane.2018.12.008.

4. Ivanusic J, Konishi $Y$, Barrington MJ. A Cadaveric study investigating the mechanism of action of erector spinae blockade. Reg Anesth Pain Med 2018; 43: 567-71.
5. Vidal E, Giménez H, Forero M, Fajardo M. Erector spinae plane block: a cadaver study to determine its mechanism of action (Spanish). Rev Esp Anestesiol Reanim 2018; 65: 514-9. 